\title{
Orbitofrontal Cortex Encodes Willingness to Pay in Everyday Economic Transactions
}

\author{
Hilke Plassmann, John O'Doherty, and Antonio Rangel \\ California Institute of Technology, Division of Humanities and Social Sciences, MC 228-77, Pasadena, California 91125
}

\begin{abstract}
An essential component of every economic transaction is a willingness-to-pay (WTP) computation in which buyers calculate the maximum amount of financial resources that they are willing to give up in exchange for the object being sold. Despite its pervasiveness, little is known about how the brain makes this computation. We investigated the neural basis of the WTP computation by scanning hungry subjects' brains using functional magnetic resonance imaging while they placed real bids for the right to eat different foods. We found that activity in the medial orbitofrontal cortex and in the dorsolateral prefrontal cortex encodes subjects' WTP for the items. Our results support the hypothesis that the medial orbitofrontal cortex encodes the value of goals in decision making.
\end{abstract}

Key words: decision making; reward; neuroeconomics; orbitofrontal cortex; fMRI; buying

\section{Introduction}

An essential component of every marketplace transaction is a willingness-to-pay (WTP) computation in which buyers calculate the maximum amount of resources that they are willing to give up in exchange for the object being sold. The WTP computation is used to evaluate whether a proposed trade is beneficial (e.g., when the WTP exceeds the price at which the item is being offered) or to decide how much to bid for an item (e.g., when competing with other individuals at an auction). To make good trades, individuals must be able to assign a WTP to an item that is commensurate to the benefits that it will generate. Otherwise they would end up purchasing items for a price that exceeds their worth to them. Despite its pervasiveness and importance for economic well being, little is known about how the brain performs the WTP computation in everyday transactions, or about how its ability to do so is affected by diseases such as addiction or obsessive compulsive disorders. This makes understanding how and where the brain makes these computations one of the most important open questions in the nascent field of neuroeconomics (Glimcher and Rustichini, 2004; Camerer et al., 2005).

Based on the results of several previous studies, we hypothesized that activity in the medial orbitofrontal cortex (mOFC) encodes WTP. Monkey electrophysiology studies of binary choice have found that activity in the OFC encodes the value of the available actions (Wallis and Miller, 2003; Padoa-Schioppa and Assad, 2006). Using functional magnetic resonance imaging (fMRI), Paulus and Frank (2003) found greater medial OFC activation during hypothetical choices than during a visual discrim-

Received May 9, 2007; revised July 2, 2007; accepted July 24, 2007.

This work was supported by the Moore Foundation, National Science Foundation Grant SES-0134618, and German Academic Exchange Service Grant DAAD D/05/47698. We thank Vivian Valentin, Jan Glaescher, Alan Hampton, and Axel Linder for their help with this work.

Correspondence should be addressed to Antonio Rangel, California Institute of Technology, Division of Humanities and Social Sciences, MC 228-77, Pasadena, CA 91125. E-mail: rangel@hss.caltech.edu.

D0I:10.1523/JNEUROSCI.2131-07.2007

Copyright $\odot 2007$ Society for Neuroscience $\quad$ 0270-6474/07/279984-05\$15.00/0 ination task (see also Arana et al., 2003). Erk et al. (2002) found that mOFC activity during a hypothetical liking rating task increased with the reported attractiveness of the stimuli. Finally, a series of stimulus-outcome learning studies (in which no decisions were made) have shown that the mOFC maintains a representation of the expected reward associated with particular cues (Rolls, 1996; Schoenbaum et al., 1998; Tremblay and Schultz, 1999; Roesch and Olson, 2004). Although all of these studies suggest that the medial OFC plays a critical role in the evaluation of choices, none of them have established that activity in the medial OFC correlates with the economic computation of WTP.

We investigated the neural basis of the WTP computation by scanning hungry subjects' brains using fMRI while they placed bids for the right to eat different foods in a Becker-DeGrootMarshak auction (Becker et al., 1964). The results described below confirmed our hypothesis: we found that activity in the right medial OFC encodes subjects' WTP for items.

\section{Materials and Methods}

Subjects. Nineteen normal-weight subjects participated in the experiment (16 males, mean age, 25.45; age range, 18-46). One additional subject participated in the experiment but was excluded from the analysis because she did not understand the instructions. All subjects were righthanded, healthy, had normal or corrected-to-normal vision, had no history of psychiatric diagnoses, neurological or metabolic illnesses, and were not taking medications that interfere with the performance of fMRI. All subjects had no history of eating disorders and were screened for liking and at least occasionally eating the types of foods that we used. Subjects were told that the goal of the experiment was to study food preferences and gave written consent before participating. Caltech's institutional The review board of the California Institute of Technology (Pasadena, CA) approved the study.

Stimuli. Subjects bid on 50 different sweet and salty junk foods (e.g., chips and candy bars). We selected the foods based on pilot data to satisfy several characteristics. First, we wanted items to be highly familiar and to be sold in local convenience stores, to remove uncertainty considerations from the WTP computation as much as possible. Familiarity data collected at the end of the experiment shows that we were successful [famil- 
A

\begin{tabular}{|c|c|c|c|}
\hline $\begin{array}{l}\text { Fast for } \\
4 \text { hours }\end{array}$ & $\begin{array}{l}100 \text { bidding trials. } \\
\text { Each of } 50 \text { items receives } \\
-1 \text { free trial bid } \\
-1 \text { forced trial bid }\end{array}$ & $\begin{array}{l}\text { Select random trial } \\
\text { implement BDM auction }\end{array}$ & $\begin{array}{l}\text { Stay in lab for } 30 \mathrm{~min} \\
\text { eat prize (if applicable) }\end{array}$ \\
\hline
\end{tabular}

B

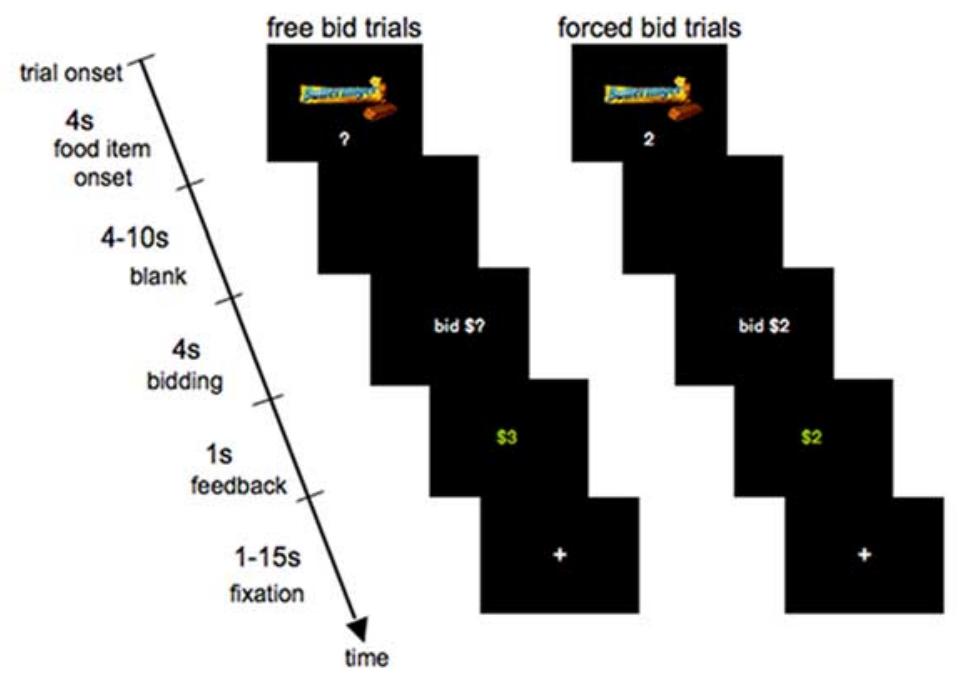

Figure 1. Experimental design. $\boldsymbol{A}$, Timeline of the experiment. $\boldsymbol{B}$, Time course for free bid and forced bid trials. Free and forced bid trials were identical except that in forced bid trials visual presentation of the food items was paired with the forced bid amount. In addition, the forced bid amount was repeated during the bidding cue. Food items, trial type, and forced bid amounts were fully randomized within subjects.

iarity scores: mean, 3.97; SD, 1.34; scale, 1 (not familiar) to 5 (very familiar)]. Second, we wanted items to be positive for the subjects (in the sense that their WTP for them is greater or equal than zero). The foods were presented to the subjects using high-resolution color pictures (72 dpi). The stimulus presentation and response recording was controlled by E-prime (Psychology Software Tools, Pittsburgh, PA). The visual stimuli were presented using video goggles.

Task. Figure 1 describes the time structure of the experiment. Subjects were instructed not to eat for $4 \mathrm{~h}$ before the experiment, which increased the value that they placed on the foods. They were also instructed that they would have to remain in the lab for $30 \mathrm{~min}$ at the conclusion of the experiment, and that the only thing that they will be able to eat is whatever food they purchased from us during the task. In addition to a \$35 participation fee, each subject received three $\$ 1$ bills in "spending money" to purchase food from us. Whatever money they did not spend was theirs to keep.

Subjects placed bids for the right to eat a snack at the end of the experiment in 100 different bidding trials. In each trial they were allowed to bid $\$ 0, \$ 1, \$ 2$, or $\$ 3$ for each food item. At the end of the experiment, one of those trials was randomly selected, by drawing a ball from an urn, and only the outcome of that trial was implemented. As a result, subjects did not have to worry about spreading their $\$ 3$ dollar budget over the different items and they could treat each trial as if it were the only decision that counted. Objects were sold using a Becker-DeGroot-Marschak auction. The rules of the auction are as follows. Let $b$ denote the bid made by the subject for a particular item. After the bid is made a random number $n$ is drawn from a known distribution (in our case, $\$ 0, \$ 1, \$ 2$, and $\$ 3$ were chosen with equal probability). If $b \geq n$, the subject got the item and paid a price equal to $n$. In contrast, if $b<n$, the subject did not get the object but also did not have to pay anything.

We used this auction institution as our model of market transactions in the laboratory because it has three very useful properties. First, it is characterized by a simple set of rules. Second, the optimal strategy for a buyer is to bid exactly her WTP for the item being sold (Becker et al., 1964). The intuition for why this is the case is as follows. There is no incentive to bid less than the WTP because the price paid is determined by the random number $n$ and, thus, the bids do not affect the price paid.
There is also no incentive to increase the bid above the WTP because this may lead to a situation in which the subject gets the item but ends up paying a price larger than his WTP (e.g., consider the case WTP $=\$ 1, b=\$ 3$, and $n=$ $\$ 2$ ). The fact that bidding the WTP is the optimal strategy was explained and emphasized extensively during the instruction and training period. We performed an extensive amount of pilot work to find a set of instructions that led to $100 \%$ reported compliance with the best strategy. The instructions, included in supplemental material (available at www.jneurosci.org), emphasized that the subject's best strategy is to look at the item, ask themselves how much is worth, and simply bid that amount. Third, because individuals always bid their exact WTP, we got a measure of the WTP computed by the brain for every bidder and item at the time of decision making, which we could then compare with the blood oxygenation level-dependent (BOLD) measure of neural activity.

To keep the task simple, subjects were only allowed to bid discrete amounts for the items $(\$ 0, \$ 1, \$ 2$, or $\$ 3)$. A consequence of this is that the bids are only approximations of the true WTP computed by subjects. For example, when the true WTP is $\$ 2.3$, our measure is $\$ 2$. Similarly, subjects with a WTP larger than $\$ 3$ enter a bid of $\$ 3$. However, the bids are a monotonic function of the true WTP and highly correlated with it.

We used two different kinds of trials: free-bid trials and forced-bid trials. Each of the 50 items was shown twice, once in a bid trial and once in a forced trial. These trials were fully randomized within and across subjects. Both types of trials had an equal probability of being selected to be the trial that counted. The timing for each type of trial is shown in Figure $1 B$. The only difference between the two types of trials is that whereas subjects were free to select the amount of their bid in the free trials, they were told how much to bid in the forced trials. The forced bids were drawn uniformly and independently from $\$ 0, \$ 1, \$ 2$, or $\$ 3$ on each trial. The set of rules described above applied to both trials. Note that subjects needed to make a willingness-to-pay computation in free trials to decide how much to bid, but they did not need to do so in forced trials.

After receiving the instructions, subjects were trained on using the response boxes with their right hand and on the bidding procedure. To avoid activation artifacts caused by the assignment of buttons to bid amounts, the assignment was counterbalanced across subjects.

The existence of two types of bidding trials is a novel and essential component of the experimental design. A difficulty in searching for the neural basis of the WTP computation is that, when the brain is exposed to a picture of a food item, it might calculate other variables that are correlated with WTP. For example, the brain may simulate the anticipated taste of the food, or it may asses its caloric content. If this issue is not properly addressed, one could erroneously attribute WTP computations to areas that are calculating different albeit correlated variables. The presence of free and forced trials provides a solution to the problem. The only difference between both types of trials is that the subject needs to perform a WTP computation in the free trials, because she needs to decide how much to bid, but not in the forced trials, because she is told what her bid should be. Every other computation, such as the anticipated taste of the food, should be performed equally in both types of trials. As a result, we can conclude that a brain area encodes the WTP computation whenever its activity increases with the WTP in the free trials, but not in the forced trials.

fMRI data acquisition. The functional imaging was conducted using a Siemens (Erlangen, Germany) 3.0 Tesla Trio MRI scanner to acquire gradient echo $\mathrm{T}^{\star}{ }^{*}$-weighted echoplanar (EPI) images with BOLD con- 
trast. To optimize functional sensitivity in the OFC, we used a tilted acquisition in an oblique orientation of $30^{\circ}$ to the anterior commissureposterior commissure line (Deichmann et al., 2003). In addition, we used an eight-channel phased array coil which yields a $40 \%$ signal increase in signal in the medial OFC over a standard head coil. Each volume comprised 32 axial slices. A total of 1100 volumes (two sessions, $\sim 18 \mathrm{~min}$ each) were collected during the experiment in an interleaved-ascending manner. The imaging parameters were as follows: echo time, $30 \mathrm{~ms}$; field of view, $192 \mathrm{~mm}$; in-plane resolution and slice thickness, $3 \mathrm{~mm}$; repetition time, $2 \mathrm{~s}$. Whole-brain high-resolution T1-weighted structural scans $(1 \times 1 \times 1 \mathrm{~mm})$ were acquired from the 19 subjects and coregistered with their mean EPI images and averaged together to permit anatomical localization of the functional activations at the group level. Image analysis was performed using SPM5 (Wellcome Department of Imaging Neuroscience, Institute of Neurology, London, UK). Temporal normalization was applied to the scans with a time of acquisition of 1.9375 referenced to the last volume. To correct for subject motion, the images were realigned to the last volume, spatially normalized to a standard $\mathrm{T} 2^{\star}$ template with a resampled voxel size of $3 \mathrm{~mm}$, and spatially smoothed using a Gaussian kernel with a full width at half maximum of $8 \mathrm{~mm}$. Intensity normalization and high-pass temporal filtering (using a filter width of $128 \mathrm{~s}$ ) were also applied to the data.

$f M R I$ data analysis. The data analysis proceeded in three steps. First, we estimated a general linear model with $\mathrm{AR}(1)$ and the following regressors that capture the main events in our experiment: free bid and picture presentation (R1), free bid and response (R2), forced bid and picture presentation (R3), forced bid and response (R4), missed bid trial and picture presentation (R5), and missed bid trial and response (R6). The regressors that capture the presentation of the food pictures were modeled using $4 \mathrm{~s}$ box-car functions. The regressors for the bid responses were modeled using stick functions.

To take advantage of the parametric nature of our design, the general linear model also included the following parametric modulators: free bid and picture presentation modulated by bid (M1), free bid and picture presentation modulated by surplus + (M2), free bid and response mod-

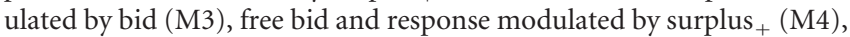
forced bid and picture presentation modulated by bid (M5), forced bid and item presentation modulated by surplus ${ }_{+}(\mathrm{M} 6)$, forced bid and item presentation modulated by surplus_ (M7), forced bid and response modulated by bid (M8), forced bid and response modulated by surplus $s_{+}$ (M9), and forced bid and response modulated by surplus _ (M10). The parametric modulators are defined as follows. "Bid" equals the amount bid for the item sold in that trial during the corresponding free trial and, thus, is a measure of the subject's WTP for the item being shown. "Surplus" is a variable that measures the expected "profit" from the trial given the bid that was placed (and conditional on the trial being selected to be the one that counts). For example, suppose that a subject's true value is $\$ 2$ and that he bids $\$ 2$. Then the surplus equals $0.25 \times \$ 2+0.25 \times \$ 1+$ $0.50 \times \$ 0=\$ 0.75$, where the first term measures the probability that the random number in the auction is 0 times the profit made in that case, the second number measure the probability that the random number is 1 times the profit made in that case, and so on. Surplus ${ }_{+}=\max [0$,surplus $]$ and Surplus $s_{-}=\min [$ surplus, 0$]$. (Note that for the free trials, the surplus variable is always non-negative, whereas for the forced trials it can be positive or negative.) We orthogonalized the modulators for each of the main regressors (M1 and M2, M3 and M4, M5 to M7, and M8 to M10). Each of the regressors was convolved with a canonical hemodynamic response function. We also included a constant term and six motion parameters as regressors of no interest. Note that the inclusion of the surplus modulator is important to avoid confounding areas that code for WTP with areas that code for economic surplus.

Second, we calculated the following first-level single-subject contrasts: (1) free-bid trials when exposed to item modulated by bid (regressor M1), (2) forced-bid trials when exposed to item modulated by bid (regressor M5), and (3) free- minus forced-bid trials when exposed to an item modulated by bid (regressors M1 minus M5).

Third, we calculated second-level group contrasts using a one-sample $t$ test. The figures shown below are constructed using these second-level contrasts at a threshold of $p<0.001$, uncorrected, and a minimum

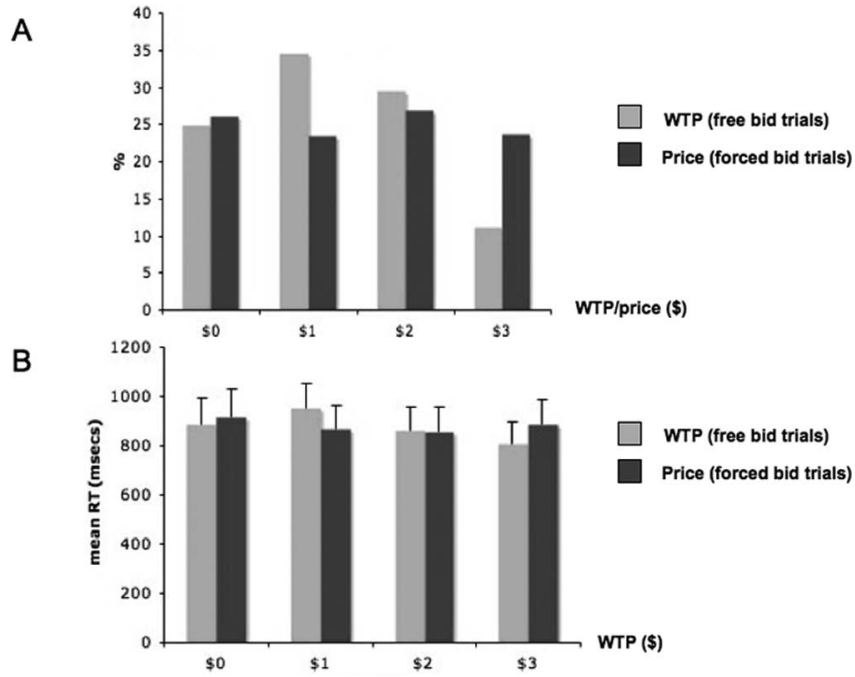

Figure 2. Behavioral results. $\boldsymbol{A}$, Distribution of bids in free and forced trials. $\boldsymbol{B}$, Reaction times for free and forced trials as a function of bid. Error bars denote SEs.

cluster size of 10. Anatomical localizations were then performed by overlaying the $t$ maps on a normalized structural image averaged across subjects, and with reference to an anatomical atlas (Duvernoy, 1999)

\section{Results}

\section{Behavioral}

Figure $2 A$ shows the distribution of bids during free- and forcedbid trials. The average free bid was $\$ 1.4$ (SD, 0.27 ) and over $75 \%$ of the free bids were greater than zero. The bid amounts for the forced bid trials were randomly drawn from a uniform distribution on $\$ 0, \$ 1, \$ 2$, and $\$ 3$. Although there is substantial variability on value that subjects place on particular items, the average WTP was significantly greater than zero $(p<0.001)$, which suggests that most items were rewarding for most subjects.

Figure $2 B$ shows the distribution of bidding reaction times for free and forced trials. The reaction times were entered in a twoway repeated-measures ANOVA with two factors: bid amount ( $\$ 0, \$ 1, \$ 2$, or $\$ 3$ ) and trial type (free- or forced-bid trial). The analysis revealed no significant main effects or interactions.

\section{Neuroimaging results}

Identifying the neural correlates of WTP in free trials

We performed a whole-brain analysis to identify areas that correlated with WTP in the free trials at the time of evaluation (i.e., when the food picture is displayed). This contrast is interesting because it identifies areas that might encode for WTP. Our hypothesis was that activity in the OFC would be positively correlated with WTP. The hypothesis was supported by the data: activity in the medial OFC $(x=6, y=30, z=-17$; $p<0.001$, uncorrected) was correlated with WTP. Other areas identified by this contrast were the dorsal anterior cingulate cortex $(x=-4$, $y=34, z=-20 ; p<0.001$, uncorrected), and the dorsolateral prefrontal cortex (DLPFC; $x=44, y=44, z=12$; $p<0.001$, uncorrected).

\section{Identifying the neural correlates of value during the forced-bid trials}

Our experimental design is based on the idea that the brain computes a WTP during free-bid trials, but not during forced-bid trials. To test this hypothesis, we performed a whole-brain analysis to identify areas correlated with WTP in the forced-bid trials at the time of evaluation (i.e., when the food picture is displayed). 

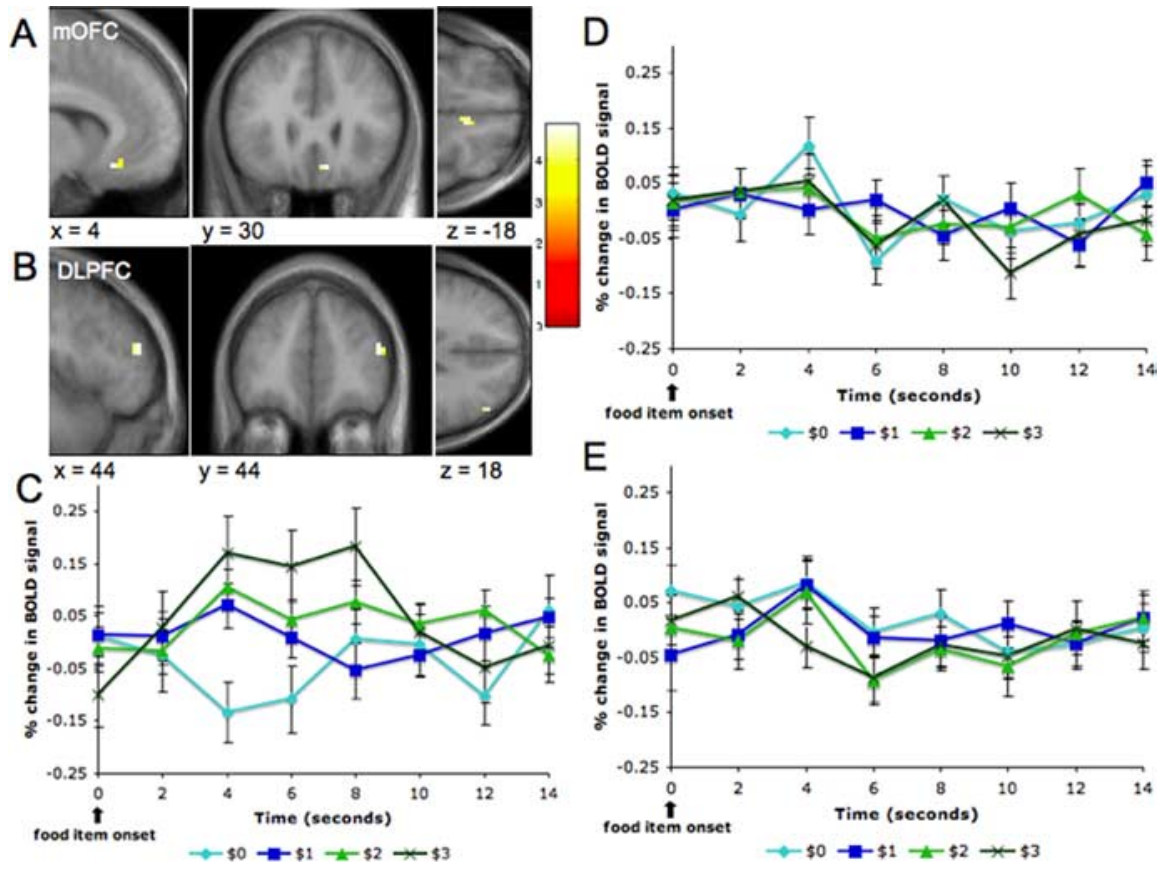

Figure 3. Neural correlates of WTP. $A, B$, Activity in the medial OFC and the DLPFC was positively correlated with WTP at the time of evaluation in the free trials more than in the forced trials. Activation maps shown at a threshold of $p<0.001$ uncorrected and 10 voxel clusters. $C$, Averaged time courses for the medial OFC voxels during free trials as a function of WTP (error bars denote SEs). D, Averaged time courses for the medial OFC voxels during forced bid trials as a function of WTP. $E$, Averaged time courses for the medial OFC voxels during forced bid trials as a function of the forced bid. A comparison of the time courses shows that the medial OFC encodes WTP in free trials, but not in forced trials, and that it does not encode the forced bid amounts.

No areas showed the desired correlation at a level of $p<0.001$ (uncorrected) and an extent threshold of 10 voxels.

Another possibility is that during the forced trials the brain encodes either the size of the forced bid, or the disagreement between the forced bid and the WTP. We tested for both possibilities using minor variants of the general linear model described above and found no regions of correlation at a level of $p<0.001$ (uncorrected) and an extent threshold of 10 voxels (for details, see the supplemental material, available at www.jneurosci.org).

\section{Test of the main hypothesis}

As discussed above, a limitation of the previous contrast is that it identifies areas with activity that is correlated with WTP, but also areas that encode for variables that are correlated with it, such as anticipatory taste. To address this potential confound, we looked for areas that (1) showed increasing activation with WTP in the free trials and (2) were significantly more activated in the free trials than in the forced trials. As predicted, we found that the right mOFC satisfied these conditions $(x=4, y=30, z=-18$; $p<0.001$, uncorrected). Unexpectedly, we also found that right DLPFC satisfied them $(x=44, y=44, z=18$; $p<0.001$, uncorrected). Figure 3, $A$ and $B$, describes the results of this contrast.

We also extracted trial averaged time-course data from peak voxels in the mOFC for each subject, which were then averaged across subjects (Fig. $3 C-E$ ). The time courses show that activity in this area during free trials showed an increase in activation that was correlated with the subjects' bids. The time courses also show that activity during forced trials did not discriminate the subjects' WTP for the items (as measured by their bids for that item during free trials) or the magnitude of the forced bids. We thus concluded that activity in the right medial OFC and dorsolateral prefrontal cortex encode for WTP in everyday economic decisions.

\section{Discussion}

In this study, we provide evidence that mOFC encodes subjects' WTP during simple economic transactions. Critically, we used a parametric experimental design that allowed us to identify areas that encode for WTP, as opposed to areas that are active during economic choice, but that do not correlate with WTP (Blair et al., 2006; Arana et al., 2003; Paulus and Frank, 2003).

Our findings are consistent with data from human lesion studies showing that lesions to the ventromedial prefrontal cortex impair the ability to make consistent pairwise choices (Fellows and Farah, 2007). Our findings are also consistent with monkey electrophysiology studies of simple choice behavior. For example, several studies have found that OFC neurons encode for the decision or incentive value of the stimuli at the time of decision making (Wallis and Miller, 2003; Roesch and Olson, 2004; Padoa-Schioppa and Assad, 2006). In addition to providing crossspecies and cross-modality validation, this experiment shows that the OFC plays a central role in the encoding of decision values in decision-making tasks that are significantly more abstract and complex that those that can be studied in monkey experiments. As a result, we speculate that the mOFC might encode for the decision value of choices in a wide class of economic settings. However, because this study and much of the previous literature has focused on the valuation of primary appetitive rewards, such as desirable foods, additional work is needed to investigate whether the $\mathrm{mOFC}$ area also encodes for the value of nonprimary rewards, such as a book or a DVD, and of negative or undesirable items, such as electric shocks of different magnitudes.

An open question in behavioral neuroscience is which parts of the OFC play a role in learning the encoding of stimulus-outcome associations (Tremblay and Schultz, 1999; Rolls, 2000) and which parts are involved in guiding decisions by encoding the value of alternative goals (Arana et al., 2003; Schoenbaum and Roesch, 2005; Feierstein et al., 2006; Padoa-Schioppa and Assad, 2006)? Our results suggest that the $\mathrm{mOFC}$ plays a critical role in goal directed behavior by encoding economic value.

We also found that activity in the DLPFC was correlated with the subject's WTP. This is consistent with previous monkey neurophysiology studies that have found neurons in the DLPFC that encode aspects of the decision or the incentive value of stimuli (Watanabe, 1996; Wallis and Miller, 2003). This raises an important open question in the neuroeconomics literature: what are the relative contributions of the DLPFC and the mOFC to the valuation of stimuli during economic decision making? The neuroanatomy of these two regions suggests a potential explanation. The OFC receives inputs from multiple sensory areas, which are likely to be used in valuation, whereas the DLPFC does not (Price, 2006). In contrast, the DLPFC is heavily connected with motor output areas, whereas the OFC is not connected with these areas directly (Petrides and Pandya, 1999). Finally, the DLPFC and the 
OFC are interconnected (Petrides and Pandya, 1999). This pattern of connectivity suggests that economic values might be first computed in the mOFC and then passed to the DLPFC to influence motor commands. This pathway is not unique as the OFC might also be able to affect actions through its connections to the striatum, which, in turn, is also heavily connected to the motor system (Yeterian and Pandya, 1991). This conjecture is consistent with our data, but our experimental design does not allow us to reach this directly.

It is interesting to compare our findings with those of Knutson et al. (2007), who study which brain areas are involved in making economic purchase decisions for unfamiliar items sold at exogenously given prices. Their task proceeds in three steps: (1) subjects are shown a picture of the item that is for sale, (2) the sale price is added, and (3) subjects decide whether to purchase the item or not. Knutson et al. (2007) obtained a measure of the value that the subjects placed on the items, albeit not a WTP, and looked for neural correlates of this value at the time of picture presentation (step 1). They found a correlation with nucleus accumbens (NAcc) activity, but not with OFC activity. In contrast, in our study activity in the medial OFC, but not in the NAcc, encoded the subject's WTP for items. Note that the difference in results is not attributable to an inability to image the NAcc because we get strong striatal activation in other contrasts of interest (supplemental Tables 3, 4, available at www.jneurosci.org as supplemental material). Instead, it is likely that there are subtle but important differences between the computations that the brain makes in the two tasks, and that the mOFC and NAcc play a differential role in such computations. For example, in the Knutson et al. (2007) task, the value of purchasing an item is the WTP minus the price and the information needed to compute this "net value" is revealed over time. In contrast, in our experiment all of the information needed to compute the WTP is revealed at the beginning of the trials. As a result, anticipatory reward signals, which are known to be correlated with NAcc activity (Knutson et al., 2001), might be computed in the Knutson et al. (2007) task, but not in the current study. Additional experiments are needed to systematically explore the differences between the computations made in the two experiments.

Part of the research agenda in neuroeconomics is to understand how the brain evaluates potential goals and outcomes at the time of decision making, and how other cognitive, emotional, and visceral processes affect the computation of economic value. A first step in this research agenda is to understand what are the brain structures responsible for the computation of value in simple everyday choices. Our results suggest that the medial OFC is a place where a variety of variables computed in other brain regions are integrated into a single representation of value. If this hypothesis is correct, other brain processes may be able to influence decision making by modulating activity in the medial OFC.

\section{References}

Arana FS, Parkinson JA, Hinton E, Holland AJ, Owen AM, Roberts AC (2003) Dissociable contributions of the human amygdala and orbito- frontal cortex to incentive motivation and goal selection. J Neurosci 23:9632-9638.

Becker G, DeGroot M, Marschak J (1964) Measuring utility by a singleresponse sequential method. Behav Sci 9:226-232.

Blair K, Marsh AA, Morton J, Vythilingam M, Jones M, Mondillo K, Pine DC, Drevets WC, Blair JR (2006) Choosing the lesser of two evils, the better of two goods: specifying the roles of ventromedial prefrontal cortex and dorsal anterior cingulate in object choice. J Neurosci 26:11379-11386.

Camerer C, Loewenstein G, Prelec D (2005) Neuroeconomics: how neuroscience can inform economics. J of Economic Literature 43:9-64.

Deichmann R, Gottfried JA, Hutton C, Turner R (2003) Optimized EPI for fMRI studies of the orbitofrontal cortex. NeuroImage 19:430-441.

Duvernoy HM (1999) The human brain: surface, three-dimensional sectional anatomy with MRI, and blood supply. Berlin: Springer.

Erk S, Spitzer M, Wunderlich A, Galley L, Walter H (2002) Cultural objects modulate reward circuitry. NeuroReport 13:2499-2503.

Feierstein CE, Quirk MC, Uchida N, Sosulski DL, Mainen ZF (2006) Representation of spatial goals in rat orbitofrontal cortex. Neuron 51:495-507.

Fellows LK, Farah MJ (2007) The role of ventromedial prefrontal cortex in decision making: judgment under uncertainty or judgment per se? Cerebral Cortex, in press.

Glimcher PW, Rustichini A (2004) Neuroeconomics: the concilience of brain and decision. Science 306:4447-4452.

Knutson B, Adams CM, Fong GW, Hommer D (2001) Anticipation of increasing monetary reward selectively recruits nucleus accumbens. J Neurosci 21:RC159.

Knutson B, Rick S, Wimmer GE, Prelec D, Loewenstein G (2007) Neural predictors of purchases. Neuron 53:147-156.

Padoa-Schioppa C, Assad JA (2006) Neurons in the orbitofrontal cortex encode economic value. Nature 441:223-226.

Paulus MP, Frank LR (2003) Ventromedial prefrontal cortex activation is critical for preference judgments. NeuroReport 14:1311-1315.

Petrides M, Pandya DN (1999) Dorsolateral prefrontal cortex: comparative cytoarchitectonic analysis in the human and the macaque brain and corticocortical connection patterns. Eur J Neurosci 11:1011-1036.

Price JL (2006) Connections of orbital cortex. In: the orbitofrontal cortex (Zald DH, Raunch SL, eds), pp 39-55. Oxford: Oxford UP.

Roesch MR, Olson CR (2004) Neuronal activity related to reward value and motivation in primate frontal cortex. Science 304:307-310.

Rolls ET (1996) The orbitofrontal cortex. Philos Trans R Soc Lond B Biol Sci 351:1433-1443, discussion 1443-1434.

Rolls ET (2000) The orbitofrontal cortex and reward. Cereb Cortex 10:284-294.

Schoenbaum G, Roesch M (2005) Orbitofrontal cortex, associative learning, and expectancies. Neuron 47:633-636.

Schoenbaum G, Chiba AA, Gallagher M (1998) Orbitofrontal cortex and basolateral amygdala encode expected outcomes during learning. Nat Neurosci 1:155-159.

Tremblay L, Schultz W (1999) Relative reward preference in primate orbitofrontal cortex. Nature 398:704-708.

Wallis JD, Miller EK (2003) Neuronal activity in primate dorsolateral and orbital prefrontal cortex during performance of a reward preference task. Eur J Neurosci 18:2069-2081.

Watanabe M (1996) Reward expectancy in primate prefrontal neurons. Nature 382:629-632.

Yeterian EH, Pandya DN (1991) Prefrontostriatal connections in relation to cortical architectonic organization in rhesus monkeys. J Comp Neurol 312:43-67. 